\title{
Correction to: Nitrogen Assimilation Varies Among Clades of Nectar- and Insect-Associated Acinetobacters
}

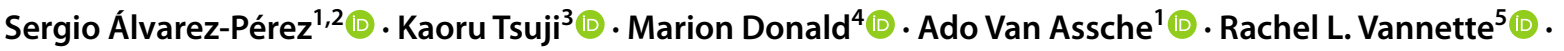 \\ Carlos M. Herrera $^{6}$ (D) Hans Jacquemyn ${ }^{7}\left[\right.$ Hadashi Fukami $^{8}\left(\mathbb{D} \cdot\right.$ Bart Lievens $^{1}{ }^{1}$
}

Published online: 17 April 2021

๑) Springer Science+Business Media, LLC, part of Springer Nature 2021

\section{Correction to: Microbial Ecology}

https://doi.org/10.1007/s00248-020-0167-x

The provisional species names Acinetobacter bareti, Acinetobacter pollinis, and Acinetobacter rathckei mentioned in the original article and its supplementary materials have been recently validated as Acinetobacter baretiae, Acinetobacter pollinis, and Acinetobacter rathckeae, respectively [Álvarez-Pérez S, et al. (2021) Acinetobacter pollinis sp. nov., Acinetobacter baretiae sp. nov. and Acinetobacter rathckeae sp. nov., isolated from floral nectar and honey bees. Int J Syst Evol Microbiol, https://doi.org/10.1099/ijsem.0. 004783].

The original article can be found online at https://doi.org/10.1007/ s00248-020-01671-x.

Sergio Álvarez-Pérez

sergioaperez@ucm.es

Bart Lievens

bart.lievens@kuleuven.be

1 Laboratory for Process Microbial Ecology and Bioinspirational Management (PME \& BIM), Department of Microbial and Molecular Systems, KU Leuven, B-3001 Leuven, Belgium

2 Department of Animal Health, Complutense University of Madrid, 28040 Madrid, Spain

3 Center for Ecological Research, Kyoto University, Hirano 2, Otsu 520-2113, Japan
4 Department of BioSciences, Rice University, Houston, TX 77005, USA

5 Department of Entomology and Nematology, University of California Davis, Davis, CA 95616, USA

6 Estación Biológica de Doñana, CSIC, 41092 Sevilla, Spain

7 Laboratory of Plant Conservation and Population Biology, Biology Department, KU Leuven, B-3001 Leuven, Belgium

8 Department of Biology, Stanford University, Stanford, CA 94305, USA 